\title{
Sur les transcendantes élémentaires et les nombres de Bernoulli et d'Euler.
}

\author{
(Par Niels Nielsex, à Copenhague.)
}

\section{PREMIÈRE PARTIE.}

\section{Applications des fonctions trigonométriques}

I. SUR Une classe de Sérues de puissances.

M. Wonprtzky $\left({ }^{*}\right)$ a donné, à l'aide de deux développements des fonctions de Bernoulli, une suite de représentations indépendantes des nombres de Bernoului et d'Euler.

Or, comme je le démontrerais dans une autre occasion $\left({ }^{* *}\right)$, les deux formules fondamentales susdites de M. Wonprazix ne sont que des représentants isolés d'une infinité de formules contenant un paramètre quelconque et donnant, pour une valeur spéciale de ce paramètre, les formules en question.

Du reste, la méthode de M. WonpıтzKx est assez partielle; car les polynomes entiers de $x$, définis comme coefficients des séries de puissances de $\alpha$, obtenues pour les fonctions

$$
(\cos \alpha)^{-x},\left(\frac{\sin \alpha}{\alpha}\right)^{-x},\left(\frac{1+e^{-\alpha}}{2}\right)^{-x},\left(\frac{1-e^{-\alpha}}{\alpha}\right)^{-x}
$$

(*) Journal de Crelle, t. 94, p. 203-232; 1883.

(**) Dans un Mémoire qui paraîtra dans les Annales de l'École Normale. 
nous donnent aussi, pour des valeurs spéciales de $x$, les nombres de BERNOuLLI et d'Euler.

Dans nos recherches suivantes nous avons à donner un nombre de représentations indépendantes des polynomes susdites et par conséquent des nombres de BeRnoulli et d'Euler.

Quant aux séries de puissances obtenues pour les fonctions (1), soit $p(x)$ une fonction analytique, régulière aux environs du point $\alpha=0$, el telle que $\varphi(0)=1$, il existe un nombre positif $\rho$, de sorte que $\varphi(\alpha)$ est, pour $|\alpha|<p$, régulière et différente de zéro.

Soit ensuite $x$ un nombre complexe quelconque, la série de puissances

$$
(\varphi(\alpha))^{-x}=\sum_{n=0}^{n=\infty} \frac{f_{n}(x) \alpha^{n}}{n !}
$$

a son rayon de convergence plus grand que zéro; de plus, il existe un nombre positif $r$, de sorte que la série (2) est uniformément convergente et par rapport à $x$ et par rapport à $\alpha$, pourvu que $|\alpha| \leqq r$ et $|x| \leqq K<\infty$.

En se rappelant les identités

$$
f_{0}(x)=1, \quad f_{n}(x)=D_{\alpha}^{n}\left[(\varphi(x))^{-x}\right]_{x=0},
$$

on conclut que $f_{n}(x)$ est un polynome entier de $x$, dont le degré ne peut jamais être plus grand que $n$.

Posons maintenant, dans (2), $y$ au lieu de $x$, puis multiplions d'après la règle de Gauchy les deux séries de puissances ainsi obtenues, nous aurons, pour les polynomes $f_{n}(x)$, la formule d'addition suivante

$$
f_{n}(x+y)=\sum_{s=1}^{s=n}\left(\begin{array}{l}
n \\
s
\end{array}\right) f_{n-s}(x) f_{s}(y) \text {. }
$$

Quant aux coefficients des polynomes $f_{n}(x)$, différentions $p$ fois par rapport à $x$ la formule (2), puis posons $x=0$, nous aurons

$$
(-1)^{n}(\log \varphi(\alpha))^{n}=\sum_{n=p}^{n=\infty} \frac{f_{n}^{(p)}(0)}{n !} \alpha^{n},
$$

formule qui montrera clairement que les polynomes $f_{n}(x)$ sont généralement d'une nature très compliquée.

Posons dans (4) $p=1$, puis différentions par rapport à $\alpha$, il résulte

$$
-\frac{\varphi^{\prime}(\alpha)}{\varphi(\alpha)}=\sum_{n=1}^{n=\infty} \frac{f^{\prime}(0)}{(n-1) !} \alpha^{n-1}
$$


différentions ensuite par rapport à $x$ la formule $(\stackrel{(2)}{)}$, ce qui donnera

$$
-x(\varphi(x))^{-x} \cdot \frac{\varphi^{\prime}(\alpha)}{\varphi(\alpha)}=\sum_{n=1}^{n=\infty} \frac{f_{n}(x)}{(n-1) !} \alpha^{n-1},
$$

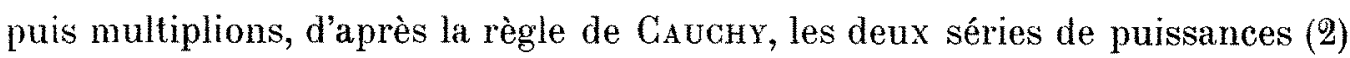
et (5), nous aurons pour les polynomes $f_{n}(x)$ les formules récursives

$$
f_{n}(x)=x \cdot \sum_{s=0}^{s=n-1}\left(\begin{array}{c}
n-1 \\
s
\end{array}\right) f_{n-s}^{\prime}(0) f_{s}(x) .
$$

Prenons maintenant pour point de départ l'identité évidente

$$
q(\alpha)=1-(1-\varphi(\alpha))
$$

puis remarquons qu'il existe un nombre positif $a$, de sorte que nous aurons, pour $|\alpha|<\sigma$, constamment $|1-\varphi(\alpha)|<1$, la formule binomiale donnera

$$
(\varphi(\alpha))^{+x}=\sum_{n=0}^{n=\infty}\left(\begin{array}{c}
x+n-1 \\
n
\end{array}\right)(1-\varphi(\alpha))^{n}
$$

où la série qui figure au secortd membre est uniformément convergente et par rapport à $x$ et par rapport à $\alpha$, pourvu que nous ayons à la fois $|x| \leqq K<\infty,|\alpha| \leqq \sigma-\delta$, où $\delta$ désigne une quantité positive arbitrairement petite.

Cela posé, nous aurons, pour les polynomes $f_{n}(x)$, les représentations indépendantes

$$
f_{n}(x)=\sum_{p=1}^{p=n}\left(\begin{array}{c}
x+p-1 \\
p
\end{array}\right) D_{\alpha}^{n}\left((1-\varphi(\alpha))^{p}\right)_{\alpha=0} .
$$

Or, il faut bien remarquer que des représentations indépendantes de la forme (8) ne sont pas particulières pour les polynomes définis à l'aide d'une série de puissances de la forme (2).

En effet, soit $n$ un positif entier et $p$ un nombre entier, tel que $0 \leqq p \leqq n$, nous aurons par décomposition (en posant $s^{0}=1$, pour $s=0$ ):

soit ensuite

$$
\frac{n ! x^{\prime \prime}}{x(x+1) \ldots(x+n)}=\sum_{s=0}^{s=n} \frac{(-1)^{s}(-s)^{n}}{x+s}\left(\begin{array}{l}
n \\
s
\end{array}\right)
$$

$$
f(x)=a_{0} x^{n}+a_{1} x^{n-1}+\cdots+a_{n-1} x+a_{n}
$$


un polynome entier quelconque du degré $n$ par rapport à $x$, puis multiplions par $a_{n-y}$ les deux membres de (9), nous aurons

$$
f(x)=x\left(\begin{array}{c}
x+n \\
n
\end{array}\right) \cdot \sum_{s=0}^{s=n} \frac{(-1)^{s} f(-s)}{x+s}\left(\begin{array}{c}
n \\
s
\end{array}\right)
$$

ou, ce qui est la même chose,

$$
f(x)=\sum_{s=0}^{s=n}(-1)^{s}\left(\begin{array}{c}
x+s-1 \\
s
\end{array}\right)\left(\begin{array}{c}
x+n \\
n-s
\end{array}\right) f(-s) .
$$

Désignons maintenant par $\alpha, \beta$ et $x$ trois nombres complexes quelconques, par $F(x)$ un polynome entier de $x$ du degré $n$ au plus, nous aurons immédiatement, en vertu de (11), cette autre formule plus générale

$$
F(\alpha x+\beta)=\sum_{s=0}^{s=n}(-1)^{s}\left(\begin{array}{c}
x+s-1 \\
s
\end{array}\right)\left(\begin{array}{c}
x+n \\
n-s
\end{array}\right) F(\beta-s \alpha) .
$$

Nous ne nous arrêtons pas par la généralisation, à l'aide de (12), de la formule (8), ce qui ne joue aucun rôle pour nos recherches suivantes.

II. Dérivées supérieures de la fongtion $(\cos \psi)^{-0}$,

Pour étudier la première des fonctions (1) du paragraphe I, nous aurons par la conclusion ordinaire de $n$ à $n+1$ une expression de la forme

$$
D_{\varphi}^{n}\left((\cos \varphi)^{-\alpha}\right)=(\cos \varphi)^{-\alpha} \stackrel{\alpha, n}{(\operatorname{tg} \varphi)}
$$

où $\stackrel{a, n}{\Phi}(\boldsymbol{x})$ est, pour tous les $n$, un polynome entier précisément du degré $n$ et par rapport à $x$ et par rapport à $\alpha$.

Supposons ensuite que $\varphi$ ne soit pas de la forme $\left(q+\frac{1}{2}\right) \pi$, où $q$ est un nombre entier, la série de puissances

$$
\left(\frac{\cos (y+\varphi)}{\cos \varphi}\right)^{-a}=\sum_{n=0}^{n=\infty} \frac{\Phi(\operatorname{tg} \varphi)}{n !} y^{n}
$$


a son rayon de convergence égal au plus petit des nombres

$$
\left|-\varphi+\left(p+\frac{1}{2}\right) \pi\right|
$$

où $p$ désigne un entier quelconque.

Posons $\operatorname{tg} \phi=x$, nous aurons en vertu de (2)

$$
(\cos y-x \sin y)^{-\alpha}=\sum_{n=0}^{n=\infty} \frac{\Phi(x)}{n !} y^{n} .
$$

Quant aux polynomes $\stackrel{\alpha, n}{\Phi(x)}$, nous aurons sans peine, en vertu de (2) et (3), ces deux équations fonctionnelles

$$
\begin{aligned}
& \stackrel{\alpha, n+1}{\Phi(x)}=\left(1+x^{2}\right) D_{x} \stackrel{\alpha, n}{ }(x)+\alpha x \Phi^{\alpha, n}(x) \\
& \Phi(x)=x(\alpha+1)\left(1+x^{2}\right) \Phi(x)-\alpha^{\alpha+2} \Phi^{\alpha, n}(x),
\end{aligned}
$$

d'où, en posant $x=0$,

$$
\Phi \stackrel{\alpha, n}{(0)}=\left(D_{r} \stackrel{\alpha, n-1}{\Phi(x)}\right)_{x \rightarrow 0}
$$

Posons pour abréger

$$
\omega_{n}(\alpha)=\alpha(\alpha+1) \ldots(\alpha+n-1), \quad n \geqq 1,
$$

nous aurons pour les six premiers des polynomes en question les expressions suivantes

$$
\begin{aligned}
& \stackrel{\alpha, 0}{\Phi(x)}=1 \\
& \stackrel{\alpha, 1}{\Phi(x)}=\alpha x \\
& \alpha, 2 \\
& \Phi(x)=\omega_{2}(\alpha) x^{2}+\alpha \\
& \underset{\alpha, 3}{\Phi(x)}=\omega_{3}(\alpha) x^{3}+\left(3 \alpha^{2}+2 \alpha\right) x \\
& \Phi(x)=\omega_{4}(x) x^{4}+2 \omega_{2}(\alpha)(3 \alpha+4) x^{2}+\left(3 x^{2}+2 \alpha\right) \\
& \Phi, 5 \\
& \Phi(x)=\omega_{5}(\alpha) x^{5}+10 \omega_{3}(x)(\alpha+2) x^{3}+\left(15 x^{3}+30 x^{2}+16 x\right) x .
\end{aligned}
$$


Remarquons que la formule (3) donnera pour $\alpha=-1$

$$
\Phi^{-1,2 n}(x)=(-1)^{n}, \quad \Phi^{-1,2 n+1}(x)=(-1)^{n-1} \cdot x
$$

nous aurons, en vertu de la formule d'addition (3) du paragraphe I, cette équation aux différences finies par rapport à $\alpha$

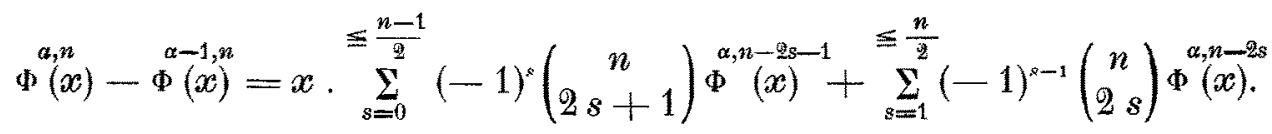

On voit que la formule générale (12) du paragraphe I ne donne aucun résultat simple pour notre fonction $\Phi$. Or, il est très facile de donner d'autres représentations indépendantes qui sont plus simples.

A cet effet, appliquons l'identité évidente

$$
\frac{\cos (y+\varphi)}{\cos \phi}=\frac{e^{i y+2 q i}+e^{-i \eta}}{e^{2 \phi i}+1}=e^{i y}\left(1+\frac{e^{-2 y i}-1}{e^{2 \varphi^{i}}+1}\right)
$$

nous aurons

$$
\left(\frac{\cos (\varphi+y)}{\cos \varphi}\right)^{-\alpha}=\sum_{p=0}^{p=\infty}(-1)^{p}\left(\begin{array}{c}
\alpha+p-1 \\
p
\end{array}\right)\left(\frac{e^{-2 y i}-1}{e^{2 \varphi^{i}}+1}\right)^{y} e^{-\alpha_{y i}},
$$

où la série ainsi obtenue est du même caractère que celle qui figure dans la formule (7) du paragraphe I.

Posons pour abréger

$$
L_{p}^{n}(\alpha)=\sum_{s=0}^{s=p}(-1)^{s}\left(\begin{array}{l}
p \\
s
\end{array}\right)(\alpha+2 p-2 s)^{n}, \quad L_{0}^{n}(\alpha)=\alpha^{n},
$$

nous aurons en vertu de (2) et en cherchant, dans la série qui figure au second membre de (9), le coefficient de la puissance $y^{n}$

$$
\Phi(\operatorname{tg} \varphi)=(-i)^{n} \sum_{p=0}^{p=n}(-1)^{p}\left(\begin{array}{c}
\alpha+p-1 \\
p
\end{array}\right) L_{p}^{n}(\alpha) \cdot \frac{e^{-i p \varphi}}{(2 \cos \varphi)^{p}},
$$

représentation indépendante qui est très curieuse.

En effet, remarquons que le premier membre de (11) est toujours réel pour des valeurs réelles de $\alpha$ et $\varphi$, nous aurons, outre les représentations indépendantes cherchées, savoir

$$
\begin{array}{r}
\alpha, 2 n \\
\Phi(\operatorname{tg} \varphi)=\sum_{p=9}^{p=2 n}(-1)^{n+p}\left(\begin{array}{c}
\alpha+p-1 \\
p
\end{array}\right) L_{p}^{2 n}(\alpha) \frac{\cos p \varphi}{(2 \cos \varphi)^{p}} \\
\Phi(\operatorname{tg} \varphi)=\sum_{p=1}^{\alpha, 2 n+1}(-1)^{n+1++1}\left(\begin{array}{c}
\alpha+p-1 \\
p
\end{array}\right) L_{p j}^{2 n+1}(\alpha) \frac{\sin p \varphi}{(2 \cos \varphi)^{p}},
\end{array}
$$


ces deux identités

$$
\begin{aligned}
& \sum_{p=0}^{p=2 n+1}(-1)^{n}\left(\begin{array}{c}
\alpha+p-1 \\
p
\end{array}\right) L_{p}^{2 n+1}(\alpha) \frac{\cos p \varphi}{(2 \cos \varphi)^{p}}=0 \\
& \sum_{p=1}^{p=2_{n}}(-1)^{n}\left(\begin{array}{c}
\alpha+p-1 \\
p
\end{array}\right) L_{p}^{2_{n}}(\alpha) \frac{\sin p \varphi}{(2 \cos \varphi)^{p}}=0 .
\end{aligned}
$$

Cela posé, les formules de Morvie

$$
\begin{aligned}
& \frac{\cos p \varphi}{(\cos \varphi)^{p}}=\sum_{s=0}^{\leqq \frac{p}{2}}(-1)^{s}\left(\begin{array}{c}
p \\
2 s
\end{array}\right)(\operatorname{tg} \varphi)^{2 s} \\
& \frac{\sin p \varphi}{(\cos \varphi)^{p}}=\sum_{s=0}^{\frac{p-1}{2}}(-1)^{s}\left(\begin{array}{c}
p \\
2 s+1
\end{array}\right)(\operatorname{tg} \varphi)^{2 s+1}
\end{aligned}
$$

donnent finalement, pour le polynome $\stackrel{\alpha, n}{\Phi}(x)$, les représentations indépendantes

$$
\begin{aligned}
& \Phi(x)=\sum_{p=0}^{\alpha=2 n}(-1)^{n+p}\left(\begin{array}{c}
\alpha+p-1 \\
p
\end{array}\right) L_{p}^{2 n}(\alpha) \frac{(1+i x)^{p}+(1-i x)^{p}}{2^{p+1}} \\
& \Phi(x)=i \cdot \sum_{p=1}^{\alpha, 2 n+1}(-1)^{n+p}\left(\begin{array}{c}
\alpha+p-1 \\
p
\end{array}\right) L_{p}^{2 n+1}(x) \frac{(1+i x)^{n}-(1-i x)^{p}}{2^{p+1}} .
\end{aligned}
$$

Remarquons que la formule (1) donnera pour $\alpha=1, \alpha=2$ respectivement

$$
\begin{aligned}
& D_{\varphi}^{n}\left(\frac{1}{\cos \varphi}\right)=\frac{1}{\cos \varphi} \stackrel{1, n}{\Phi}(\operatorname{tg} \varphi) \\
& D_{\varphi}^{n+1} \operatorname{tg} \varphi=\left(1+\operatorname{tg}^{2} \varphi\right) \stackrel{2, n}{\Phi}(\operatorname{tg} \varphi),
\end{aligned}
$$

nous venons de donner des représentations indépendantes de ces deux dérivées d'ordre supérieur.

M. JENSEN a trouvé, il y a trente ans à peu près, de telles représentations indépendantes, et un grand nombre d'autres formules, à l'aide du calcul symbolique; mais malheureusement il n'a pas publié les très beaux et très importants résultats qu'il a trouvés par ce procédé $\left({ }^{*}\right)$.

(*) Dans une conférence, faite à la Société Mathématique de Copenhague dans la séance du 25 janvier 1912, M. JENSEN a développé les plus importants de ses résultats susdits. 
III. ÉtTUDE DES FONGTIONS PARTICULIÈRES.

Posons dans la formule (3) du paragraphe II $x=0, \alpha=x$, nous avons à étudier les polynomes

$$
a_{n}(x)=\Phi(0)=\left(D_{\alpha} \stackrel{x, 2,2 n-1}{\Phi}(\alpha)\right)_{\alpha=0}, \quad n \geqq 1
$$

ce qui nous conduira à la série de puissances

$$
(\cos x)^{-x}=\sum_{n=0}^{n=\infty} \frac{a_{n}(x)}{(2 n) !} \alpha^{2 n}, \quad|\alpha|<\frac{\pi}{2} .
$$

L'équation fonctionnelle (5) du paragraphe II donnera dans ce cas

$$
a_{n+1}(x)=x(x+1) a_{n}(x+2)-x^{2} a_{n}(x),
$$

d'où particulièrement

$$
a_{n+1}^{\prime}(0)=a_{n}(2), \quad a_{n}(-1)=(-1)^{n},
$$

de sorte que les séries de puissances

$$
\begin{aligned}
& \frac{1}{\cos \alpha}=1+\sum_{n=1}^{n=\infty} \frac{E_{n} \alpha^{2 n}}{(2 n) !}, \quad|\alpha|<\frac{\pi}{2} \\
& \operatorname{tg} \alpha=\sum_{n=0}^{n=\infty} \frac{T_{n+1} \alpha^{2 n+1}}{(2 n+1) !}, \quad|\alpha|<\frac{\pi}{2},
\end{aligned}
$$

où les $E_{n}$ sont les nombres d'EuLER, les $T_{n}$ les coefficients des tangentes, donnent ces valeurs spéciales

$$
a_{n}^{\prime}(0)=T_{n}, \quad a_{n}(1)=E_{n}, \quad a_{n}(2)=T_{n+1} .
$$

Appliquons ensuite les formules (3) et (6) du paragraphe I, nous aurons ici

$$
\begin{array}{r}
a_{n}(x+y)=\sum_{s=0}^{s=n}\left(\begin{array}{ll}
2 & n \\
2 & s
\end{array}\right) a_{n-s}(x) a_{s}(y) \\
a_{n+1}(x)=x \cdot \sum_{s=1}^{s+1}\left(\begin{array}{l}
2 n+1 \\
2 s-1
\end{array}\right) T_{s} a_{n-s+1}(x),
\end{array}
$$

ce qui domnera pour les six premiers des polynomes $a_{n}(x)$ les expressions 
suivantes

$$
\begin{aligned}
& a_{0}(x)=1 \\
& a_{1}(x)=x \\
& a_{2}(x)=3 x^{2}+2 x \\
& a_{3}(x)=15 x^{3}+30 x^{2}+16 x \\
& a_{4}(x)=105 x^{4}+420 x^{3}+588 x^{2}+272 x \\
& a_{3}(x)=945 x^{5}+6300 x^{4}+16380 x^{3}+18960 x^{2}+7936 x
\end{aligned}
$$

Nous avons encore à déterminer le nombre entier $a_{n}(-p)$, où $p>1$ est un entier.

A cet effet, nous appliquons la formule élémentaire

$$
(\cos \alpha)^{p}=\frac{1}{2^{p}} \cdot \sum_{s=0}^{\leqq \frac{p}{2}}\left(\begin{array}{l}
p \\
s
\end{array}\right) \varepsilon_{p-2 s} \cos (p-2 s) \alpha,
$$

où il faut admettre $\varepsilon_{0}=1$, mais $\varepsilon_{m}=2$ pour $m \geqq 1$. Posons pour abréger

$$
\mathfrak{A}_{p}^{*}=\sum_{s=0}^{\leqq \frac{p-1}{2}}\left(\begin{array}{c}
p \\
s
\end{array}\right)(p-2 s)^{n},
$$

nous aurons

$$
D_{a}^{2 \pi}\left(\cos ^{p} \alpha\right)_{\alpha_{0=0}}=\frac{(-1)^{n}}{2^{p-1}} \mathfrak{H}_{p}^{2 n}
$$

d'où la valeur cherchée

$$
a_{n}(-p)=\frac{(-1)^{n}}{2^{p-1}} \mathfrak{A}_{p}^{9_{n}^{n}}, \quad u \geqq 1
$$

Cela posé appliquons, pour $\beta=0, \alpha=q$, où $q$ désigne un positif entier, la formule générale (12) du paragraphe I, nous aurons pour $\alpha_{n}(x)$ la représentation indépendante

$$
a_{n}(x)=\sum_{p=1}^{p=n} \frac{(-1)^{n+r}}{2^{q p-1}}\left(\begin{array}{c}
\frac{x}{q}+p-1 \\
p
\end{array}\right)\left(\begin{array}{c}
\frac{x}{q}+n \\
n-p
\end{array}\right)_{\mathfrak{A}_{p q}^{2 n},}
$$

d'où particulièrement pour $q=1$

$$
a_{n}(x)=\sum_{p=1}^{p=i n} \frac{(-1)^{n+p}}{2^{p-1}}\left(\begin{array}{c}
x+p-1 \\
p
\end{array}\right)\left(\begin{array}{c}
x+n \\
n-p
\end{array}\right) \mathfrak{A}_{p}^{2 n}
$$

Combinons maintenant les formules (1) et (12), (13) du paragraphe II, 
nous aurons, pour $a_{n}(x)$, ces deux autres represéntations indépendantes

$$
\begin{gathered}
a_{n}(x)=\sum_{p=0}^{p=2 n} \frac{(-1)^{n+p}}{2^{p}}\left(\begin{array}{c}
x+p-1 \\
p
\end{array}\right) L_{p}^{2 n}(x) \\
a_{n}(x)=\sum_{p=1}^{p=2 n-1} \frac{(-1)^{n+p} p}{2^{p}}\left(\begin{array}{c}
x+p-1 \\
p
\end{array}\right) L_{p}^{2 n-1}(x) .
\end{gathered}
$$

D'autres représentations indépendantes peuvent être déduites à l'aide des identités

$$
\cos \alpha=1-2 \sin ^{2} \frac{\alpha}{2}=\left(1-\sin ^{2} \alpha\right)^{\frac{1}{2}}
$$

ce qui donnera

$$
(\cos \alpha)^{-x}=\sum_{p=0}^{p=\infty}\left(\begin{array}{c}
x+p-1 \\
p
\end{array}\right)\left(2 \sin ^{2} \frac{\alpha}{2}\right)^{p}=\sum_{p=0}^{p=\infty}\left(\begin{array}{c}
\frac{x}{2}+p-1 \\
p
\end{array}\right)(\sin \alpha)^{2 p},
$$

de sorte que nous avons à calculer les dérivées

$$
D_{\alpha}^{n}\left((\sin \alpha)^{n}\right)_{\alpha=0}
$$

où $n$ et $p$ sont des positifs entiers.

A cet effet, appliquons les formules élémentaires

$$
\begin{aligned}
& (\sin \alpha)^{2 p}=\frac{(-1)^{p}}{2^{2 p}} \sum_{s=0}^{s=p}(-1)^{s}\left(\begin{array}{c}
2 p \\
s
\end{array}\right) \varepsilon_{2 p-2 s} \cos (2 p-2 s) \alpha \\
& (\sin \alpha)^{2 p+1}=\frac{(-1)^{s}}{2^{2 p}} \sum_{s=0}^{s=p}(-1)^{s}\left(\begin{array}{c}
2 p+1 \\
s
\end{array}\right) \sin (2 p-2 s+1) \alpha
\end{aligned}
$$

puis posons pour abréger

$$
A_{p}^{n}=\sum_{s=0}^{\leq \frac{p-1}{2}}(-1)^{s}\left(\begin{array}{l}
p \\
s
\end{array}\right)(p-2 s)^{n}
$$

nous aurons

$$
D_{\alpha}^{n}\left(\sin ^{p} \alpha\right)_{\alpha=0}=\frac{(-1)^{q}}{2^{p-1}} A_{p}^{n}, \quad n-p=2 q \geqq 0
$$

Cela posé, nous aurons, en vertu de (14), ces deux autres représentations 
indépendantes

$$
\begin{aligned}
& a_{n}(x)=\sum_{p=1}^{p=n} \frac{(-1)^{n+p}}{2^{2 n+p-1}}\left(\begin{array}{c}
x+p-1 \\
p
\end{array}\right) A_{2 p}^{2 n} \\
& a_{n}(x)=\sum_{p=1}^{p=n} \frac{(-1)^{n+p}}{2^{2 p-1}}\left(\begin{array}{c}
\frac{x}{2}+p-1 \\
p
\end{array}\right) A_{2 p}^{2 n} .
\end{aligned}
$$

Étudions encore la série de puissances

$$
\left(\frac{\sin \alpha}{\alpha}\right)^{-x}=\sum_{n=0}^{n=\infty} \frac{b_{n}(x)}{(2 n) !} \alpha^{2 n}, \quad|\alpha|<\pi,
$$

les formules d'EuLER

$$
\begin{aligned}
& \frac{1}{\alpha}-\cot \alpha=\sum_{n=1}^{n=\infty} \frac{2^{2 n} B_{n}}{(2 n) !} \alpha^{3 n-1},|\alpha|<\pi, \\
& \left(\frac{\alpha}{\sin \alpha}\right)^{2}=1+\sum_{n=1}^{n=\infty} \frac{2^{2 n}(2 n-1) B_{n}}{(2 n) !} \alpha^{2 n}, \quad|\alpha|<\pi,
\end{aligned}
$$

où les $B_{n}$ sont les nombres de Bernoulu, donneront

$$
b_{n}^{\prime}(0)=\frac{2^{2 n} B_{n}}{2 n}, \quad b_{n}(1)=\left(2^{2 n}-2\right) B_{n}, \quad b_{n}(2)=(2 n-1) 2^{2 n} B_{n} .
$$

De plus, nous trouvons en vertu de (16)

$$
b_{n}(-p)=\frac{(-1)^{n}(2 n) !}{(p+2 n) ! 2^{p-1}} A_{p}^{p+2 n}, \quad p \geqq 1,
$$

ce qui donnera, en vertu de la formule générale (12) du paragraphe I, cette représentation indépendante

$$
b_{n}(x)=\sum_{p=1}^{p=n}(-1)^{n+p}\left(\begin{array}{c}
\frac{x}{q}+p-1 \\
p
\end{array}\right)\left(\begin{array}{c}
\frac{x}{q}+n \\
n-p
\end{array}\right) \frac{(2 n) !}{(p q+2 n) ! 2^{p q-1}} A_{p q}^{p q+2 n}
$$

où $q$ désigne un positif entier quelconque. 


\section{DEUXIÈME PARTIE.}

\section{Applications de la fonction exponentielle}

\section{Généralisation d'Une sérif d'Euler.}

Soient $x$ et $\alpha$ deux nombres donnés quelconques, il existe un nombre positif $\rho$, tel que la série de puissances de $y$

$$
\left(\frac{x-e^{-y(x-1)}}{x-1}\right)^{-a}=\sum_{n=0}^{n=\infty} \frac{(-1)^{n} \Psi^{\alpha, n}(x)}{n !} y^{n}
$$

a son rayon de convergence égal à $\rho$; pour les coefficients $\Psi^{\alpha, n}(x)$ nous trouvons sans peine les équations fonctionnelles

$$
\begin{aligned}
& \stackrel{\alpha, n+1}{\Psi}(x)=\alpha \cdot \stackrel{\alpha+1, n}{\Psi}(x)-\alpha(x-1) \stackrel{\alpha, n}{\Psi}(x) \\
& \underset{\alpha, n+1}{\Psi}(x)=(\alpha+n x) \stackrel{\alpha, n}{\Psi}(x)-x(x-1) D_{x} \Psi^{\alpha, n}(x),
\end{aligned}
$$

d'où nous obtenons pour les six premières fonctions $\Psi^{a, n}(x)$ les expressions suivantes

$$
\begin{aligned}
& \Psi(x)=1 \\
& \stackrel{\alpha, 1}{\Psi(x)}=\alpha \\
& \stackrel{\alpha, 2}{\Psi}(x)=\alpha x+\alpha^{2} \\
& \Psi(x)=\alpha x^{2}+\left(3 \alpha^{2}+\alpha\right) x+\alpha^{3} \\
& \stackrel{\alpha, 4}{\Psi}(x)=\alpha x^{3}+\left(7 \alpha^{2}+4 \alpha\right) x^{2}+\left(6 \alpha^{3}+4 \alpha^{2}+\alpha\right) x+\alpha^{4} \\
& \Psi(x)=\alpha x^{4}+\left(15 \alpha^{2}+11 \alpha\right) x^{3}+\left(25 x^{3}+30 \alpha^{2}+11 \alpha\right) x^{2}+ \\
& +\left(10 \alpha^{4}+10 \alpha^{3}+5 \alpha^{2}+\alpha\right) x+\alpha^{5} \text {. }
\end{aligned}
$$


Combinons ces valeurs spéciales et les équations fonctionnelles susdites, nous verrons que $\stackrel{\alpha, n}{(x)}$ est un polynome entier et par rapport à $\alpha$ et par rapport à $x$, et ce polynome est du degré $n$ par rapport à $\%$ mais du degré $n-1$ par rapport à $x$; dans le dernier cas il faut supposer $n \geqq 1$.

Posons $\alpha=-1$, nous aurons

$$
\Psi^{-1, n}(x)=-(x-1)^{n-1}, \quad n \geqq 1,
$$

ce qui donnera, en vertu de la formule d'addition (3) du paragraphe I, l'écuation aux différences finies

$$
\Psi(x)=\stackrel{\alpha-1, n}{\alpha, n}+\sum_{s=1}^{s=n}\left(\begin{array}{l}
n \\
s
\end{array}\right)(x-1)^{r \cdots+1} \Psi^{\alpha, j i-s}(x) .
$$

La même formule d'addition domnera, en verhu de (2), cette autre formuls: spéciale

$$
\Psi^{\alpha}(x)=x \cdot \Psi^{\alpha, n}(x)+\alpha x \cdot \sum_{s=1}^{n=n}\left(\begin{array}{l}
n ! \\
s
\end{array}\right) \Psi^{\alpha, n-s}(x) \Psi \Psi^{1, s}(x)
$$

qui nous sera très utile dans ce qui suit.

Il est très facile de trourer, pour les $\Psi^{\alpha, n}(x)$, des représentations indépendantes; car l'identité évidente

$$
\frac{x-e^{-y(x-3)}}{x-1}=1-\frac{e^{-y(x-1)}-1}{x-1}
$$

donnera la formule

$$
\left(\frac{x-e^{-n(x-1)}}{x-1}\right)^{-\alpha}=\sum_{n=0}^{n:=\infty}\left(\begin{array}{c}
x+n-1 \\
n
\end{array}\right)\left(\frac{e^{-x(x-1)}-1}{x-1}\right)^{\prime}
$$

qui est du même caractère que la formule (7) du paragraphe I.

Appliquons ensuite la formule, où $v$ est un positif entier,

$$
\left(e^{-y(x-1)}-1\right)^{p}=\sum_{s=0}^{s=p}(-1)^{s}\left(\begin{array}{c}
p \\
s
\end{array}\right) e^{-(p-s) \psi(x-1)},
$$

puis posons pour abréger

$$
L_{p}^{n}=\sum_{r=0}^{r=p-1}(-1)^{r}\left(\begin{array}{l}
p \\
r
\end{array}\right)(p-r)^{n}=\frac{1}{2^{n}} L_{p}^{n}(0),
$$

où $L_{i j}^{n}(\alpha)$ est la fonction définie par la formule (10) du paragraphe II, nous 
aurons

$$
\left(e^{-y(x-1)}-1\right)^{p}=\sum_{n=p}^{n=\infty} \frac{(-1)^{n} L_{p}^{n}}{n !} y^{n}(x-1)^{n},
$$

d'où, en vertu de (1) et (6), la représentation suivante

$$
\Psi(x)=\sum_{p=1}^{\alpha=n}\left(\begin{array}{c}
\alpha+p-1 \\
p
\end{array}\right) L_{p}^{n} \cdot(x-1)^{n-p} .
$$

Ordonnons maintenant, selon des puissances ascendantes de $x$, le second membre de (8), puis posons

nous aurons

$$
\Psi(x)=\sum_{p=1}^{\alpha=n} \sum_{p}^{n}(\alpha) x^{n-\mu},
$$

$$
\mathfrak{R}_{p}^{n}(\alpha)=\sum_{r=0}^{r=p-1}(-1)^{r}\left(\begin{array}{c}
n-p+r \\
p-r
\end{array}\right)\left(\begin{array}{c}
x+p-r-1 \\
p-r
\end{array}\right) L_{p-r}^{n} .
$$

Cherchons ensuite, au second membre de (10), le coefficient de la puissance $(p-r)^{n}$, ce coefficient deviendra

$$
\begin{aligned}
\sum_{s=0}^{s=1}\left(\begin{array}{c}
\alpha+p-s-1 \\
p-s
\end{array}\right) & \left(\begin{array}{c}
n-p+s \\
s
\end{array}\right)\left(\begin{array}{c}
p-s \\
r-s
\end{array}\right)= \\
& =\left(\begin{array}{c}
\alpha+p-r-1 \\
p-r
\end{array}\right) \sum_{s=0}^{s=r}\left(\begin{array}{c}
\alpha+p-s-1 \\
r-s
\end{array}\right)\left(\begin{array}{c}
n+p-s \\
s
\end{array}\right) ;
\end{aligned}
$$

nous aurons pour $\mathfrak{\ell}_{p}^{n}(\alpha)$ cette autre expression

$$
\mathfrak{Q}_{p}^{n}(\alpha)=\sum_{r=0}^{r=p-1}(-1)^{r}\left(\begin{array}{c}
x+p-r-1 \\
p-r
\end{array}\right)\left(\begin{array}{c}
x+n \\
r
\end{array}\right)(p-r)^{\prime \prime} .
$$

Inversement nous aurons en vertu de (9) et (10)

$$
\left(\begin{array}{c}
\alpha+p-1 \\
p
\end{array}\right) L_{L^{n}}^{n}=\sum_{s=0}^{s=p-1}\left(\begin{array}{c}
n-p+s \\
s
\end{array}\right) \Re_{p-s}^{n}(*)
$$

Introduisons maintenant dans (2) et (3) les expressions (9) et (10), nous aurons les formules récursives

$$
\begin{gathered}
L_{p}^{n+1}=p\left(L_{y}^{n}+L_{p-1}^{n}\right) \\
\mathfrak{R}_{p}^{n+1}(\alpha)=p \mathfrak{R}_{p}^{n}(x)+(n-p+2) \mathfrak{R}_{p-1}^{n}(\alpha),
\end{gathered}
$$

dont la première est due à Grunert (*).

(*) Mathematische Abhandlungen, Altona, 1892. Citation de M. Wonpiтzк $\mathrm{F}$, dans le Journnl de Crelle. t. 9 ', p. $210 ; 1883$. 
Posons dans (1) $x=0, x=1$, la fonction qui figure au premier membre deviendra respectivement

ce qui donnera

$$
e^{--\sigma y},(1+y)^{-\alpha},
$$

$$
\Psi^{\alpha, n}(0)=\alpha^{n}, \quad \stackrel{\alpha, 2}{\Psi}(1)=\left(\begin{array}{c}
\alpha+n-1 \\
n
\end{array}\right)
$$

d'où en vertu de (8) et (9) ces deux développements

$$
\begin{gathered}
x^{n}=\sum_{p=1}^{p=l}(-1)^{n-1}\left(\begin{array}{c}
x+p-1 \\
p
\end{array}\right) I_{p}^{n} \\
\left(\begin{array}{c}
x+n-1 \\
n
\end{array}\right)=\sum_{p=1}^{p=n} \ell_{p}^{n}(x) .
\end{gathered}
$$

La première de ces deux formules est due à CAUCHY $\left(^{*}\right)$ et joue un rôle fondamental dans les recherches de $M$. Worriтzкy.

\section{Formules d'Eulek, de Laplace ett de Sciherk.}

Le cas particulier de nos recherches précédentes qui correspond à $\alpha=1$ est classique.

Posons pour abréger

$$
\psi_{, \prime}(x)=\Psi^{1, n}(x)
$$

l'équation fonctionelle (2) du paragraphe IV donnera

$$
\left(D_{\alpha} \Psi^{\alpha, n+1}(x)\right)_{n=0}=x \psi_{n}(x), \quad n \geqq 1 ;
$$

c'est-à-dire que nous aurons ces deux séries de puissances

$$
\begin{gathered}
\frac{x-1}{x-e^{-y(x-1)}}=\sum_{n=1}^{n=\infty} \frac{(-1)^{n} \psi_{n}(x)}{n !} y^{n} \\
\log \left(\frac{x-1}{x-e^{-y(x-1)}}\right)=-y+\sum_{n=1}^{n=\infty} \frac{(-1)^{n+1} x \psi_{n}(x)}{(n+1) !} y^{n+1},
\end{gathered}
$$

(*) Résumés Analytiques, p. 35; Turin, 1833 . 
tandis que les formules (4) et (5) du paragraphe IV donnent ici

$$
\begin{gathered}
\psi_{n}(x)=\sum_{s=1}^{s=n}\left(\begin{array}{l}
n \\
s
\end{array}\right)(x-1)^{s-1} \dot{\psi}_{n-s}(x), n \geqq 1 \\
\psi_{n+1}(x)=(x+1) \psi_{n}(x)+x \cdot \sum_{s=1}^{s=n-1}\left(\begin{array}{l}
n \\
s
\end{array}\right) \psi_{s}(x) \psi_{n-s}(x) .
\end{gathered}
$$

Posons pour abréger.

$$
\mathbb{R}_{p}^{n}=\sum_{r=0}^{r=p-1}(-1)^{n}\left(\begin{array}{c}
n+1 \\
r
\end{array}\right)(p-r)^{n}=\ell_{p}^{n}(1)
$$

nous aurons ces deux représentations indépendantes

$$
\begin{aligned}
& \psi_{n}(x)=\sum_{p=1}^{p=n} L_{p}^{n}(x-1)^{n-l^{\prime}} \\
& \psi_{n}(x)=\sum_{p=1}^{p=n} \xi_{p}^{n} x^{n-p}
\end{aligned}
$$

dont la seconde est indiquée par Eulen $\left(^{*}\right)$ et démontrée par Laplace $\left(^{* *}\right)$. EULer $\left.{ }^{* * *}\right)$ a calculé les huit premiers des fonctions $\psi_{n}(x)$, savoir

$$
\begin{aligned}
& \psi_{0}(x)=1 \\
& \psi_{1}(x)=1 \\
& \psi_{2}(x)=x+1 \\
& \psi_{3}(x)=x^{2}+4 x+1 \\
& \psi_{4}(x)=x^{3}+11 x^{2}+11 x+1 \\
& \psi_{5}(x)=x^{4}+26 x^{3}+66 x^{2}+26 x+1 \\
& \psi_{6}(x)=x^{5}+57 x^{4}+302 x^{3}+302 x^{2}+57 x+1 \\
& \psi_{7}(x)=x^{6}+120 x^{5}+1191 x^{4}+2416 x^{3}+1191 x^{2}+120 x+1 .
\end{aligned}
$$

Il saute aux yeux que ces polynomes spéciaux sont réciproques; appliquons la formule récursive (5), nous aurons la proposition suivante due à Sсненк $\left({ }^{* * * *}\right)$ :

(*) Institutiones caleuli differentialis, p. 486 ; Saint-Pétersbourg, 1755 .

(**) Voir LAcrolx : Traité du calcul différentiel et intégral, t. 3, p. 110; Paris, 1819.

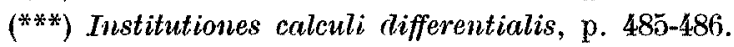

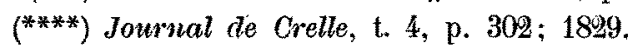


Le polynome $\psi_{n}(x)$ est, pour $n \geqq 1$, un polynome réciproque du degré $n-1$, savoir

$$
x^{n-1} \psi_{n}\left(\frac{1}{x}\right)=\psi_{n}(x)
$$

de sorte que nous aurons, pour $1 \leqq p<n$ :

$$
\mathfrak{R}_{p}^{n}=\mathfrak{R}_{n-p+1}^{n} .
$$

Remplaçons maintenant dans (2) $y$ par $y:(x-1)$, il en résulte

$$
\frac{x-1}{x-e^{-y}}=1+\sum_{n=1}^{n=\infty} \frac{(-1)^{n} \psi_{n}(x)}{n !(x-1)^{n}} y^{n}
$$

d'où en posant $-e^{2 x i}$ au lieu de $x$ et $2 h i$ au lieu de $y$

$$
\frac{1}{e^{2 x i}+e^{-2 \overline{n i}}}=\frac{1}{e^{2 x i}+1}+\sum_{n=1}^{n=\infty} \frac{\psi_{n}\left(-e^{2 x i}\right)(2 h i)^{n}}{n !\left(e^{2, n i}+1\right)^{n+1}},
$$

ce qui donnera, en vertu des identités

$$
e^{2 x i}+1=2 e^{x i} \cos x, \quad \operatorname{tg}(x+h)=\frac{1}{i}\left(\frac{2 e^{2 x i}}{e^{2 x i}+e^{-2 h i}}-1\right),
$$

la série de TaYLok

$$
\operatorname{tg}(x+h)=\operatorname{tg} x+\sum_{n=1}^{n=\infty} \frac{i^{n-1} \psi_{n}\left(-e^{2 x i}\right) e^{-(n-1) x i} h^{n}}{n !(\cos x)^{n+1}},
$$

de sorte que nous aurons en différentiant $n$ fois par rapport à $h$, puis posant $h=0$

$$
D_{x}^{n} \operatorname{tg} x=\frac{i^{n-1} \psi_{n}\left(-e^{2 x i}\right) e^{-(n-1) x i}}{(\cos x)^{n+1}}
$$

Cela posé, introduisons dans (11) le développement (7), nous aurons

$$
D_{x}^{n} \operatorname{tg} x=(-i)^{n-1} \cdot \sum_{p=0}^{p=1 n-1}(-1)^{n} 2^{n-p^{n-1}} L_{p+1}^{n} \cdot \frac{e^{-p x i}}{(\cos x)^{p+2}},
$$

ce qui donnera les deux représentations indépendantes

$$
\begin{aligned}
& D_{x}^{2 n} \operatorname{tg} x=\frac{2^{2 n-1}}{(\cos x)^{2}} \cdot \sum_{p=1}^{p=2 n-1}(-1)^{n+p} L_{p+1}^{2 n} \cdot \frac{\sin p x}{(2 \cos x)^{p}} \\
& D_{x}^{2 n+1} \operatorname{tg} x=\frac{2^{2 n}}{(\cos x)^{2}} \cdot \sum_{p=0}^{p=2 n}(-1)^{n+p} L_{p+1}^{2 n+1} \cdot \frac{\cos p x}{(2 \cos x)^{p}}
\end{aligned}
$$


et Jes identités

$$
\begin{aligned}
& \sum_{p=1}^{p=2 n n}(-1)^{p-1} L_{p+1}^{2 n+1} \cdot \frac{\sin p x}{(2 \cos x)^{p}}=0 \\
& \sum_{p=0}^{2 n-1}(-1)^{p} L_{p+1}^{2 n} \cdot \frac{\cos p x}{(2 \cos x)^{p}}=0 .
\end{aligned}
$$

On voit que ces formules sont du même genre que les formules plus générales (12), (13) et (14), (15) développées dans le paragraphe II, mais que les coefficients ne sont pas formellement les mêmes. Nous ne nous arrêtons pas à l'étude des relations numériques ainsi obtenues.

Introduisons ensuite dans (11) le développement (8), nous trouvons les formules de Schrrk $\left(^{*}\right)$

$$
\begin{gathered}
D_{x}^{2 n} \operatorname{tg} x=2 \cdot \sum_{p=0}^{p=n-1}(-1)^{p} \mathbb{R}_{n-p}^{2 n} \cdot \frac{\sin (2 p+1) x}{(\cos x)^{2 n+1}} \\
D_{x}^{2 n+1} \operatorname{tg} x=\frac{\mathbb{R}_{n+1}^{2 n+1}}{(\cos x)^{2 n+2}}-2 \cdot \sum_{p=0}^{p=n-1}(-1)^{p} \mathbb{R}_{n-p}^{2 n+1} \cdot \frac{\cos (2 p+2) x}{(\cos x)^{2 n+2}} .
\end{gathered}
$$

VI. Étude d'autres Fonctions particulières.

Posons dans les formules du paragraphe IV $x=-1$ et $x$ au lieu de $\alpha$, nous avons à étudier les fonctions

$$
c_{n}(x)=\Psi(-1)
$$

ce qui nous conduira à la série de puissances

$$
\left(\frac{1+e^{2 \alpha}}{2}\right)^{-x}=\sum_{n=0}^{n=\infty} \frac{(-1)^{n} c_{n}(x)}{n !} x^{n}, \quad|\alpha|<\frac{\pi}{2} .
$$

Dans ce cas nous aurons l'équation fonctionnelle

$$
c_{n+1}(x)=2 x c_{n}(x)-x c_{n}(x+1)
$$

et les représentations indépendantes

$$
c_{n}(x)=\sum_{p=1}^{p=i t}(-2)^{n-p}\left(\begin{array}{c}
x+p-1 \\
p
\end{array}\right) L_{p}^{*}
$$

(*) Jourual ae Crelle, t. 4 , p. 303; 1829. 


$$
c_{n}(x)=\sum_{p=1}^{p=n}(-1)^{n-p} \Re_{p}^{n}(x)
$$

ce qui domnera les valeurs spéciales

$$
\begin{aligned}
& c_{0}(x)=1 \\
& c_{1}(x)=x \\
& c_{2}(x)=x^{2}-x \\
& c_{3}(x)=x^{3}-3 x^{2} \\
& c_{4}(x)=x^{4}-6 x^{3}+3 x^{2}+2 x \\
& c_{5}(x)=x^{5}-10 x^{4}+15 x^{3}+10 x^{2} .
\end{aligned}
$$

Remarquons que l'équation fonctionelle (3) dommera

$$
c_{n+1}^{\prime}(0)=-c_{n}(1), \quad c_{n}(2)=2 c_{n}(1)-c_{n+1}(1),
$$

nous aurons, en vertu de la série de puissances

$$
\frac{2}{1+e^{2 m}}=1+\sum_{n=1}^{n=\infty} \frac{(-1)^{n} T_{n}}{(2 n-1) !} \alpha^{2 n-1}, \quad(x)<\frac{\pi}{2},
$$

ces valeurs numériques

$$
\begin{aligned}
& c_{2 n}^{\prime}(0)=(-1)^{n} T_{n}, \quad c_{2 n+1}^{\prime}(0)=0 \\
& c_{2 n}(1)=0, \quad c_{2 n+1}(1)=(-1)^{n} T_{n+1}^{\prime} \\
& c_{2 n}(2)=(-1)^{n+1} T_{n+1}, \quad c_{2 n+1}(2)=(-1)^{n} 2 T_{n+1} .
\end{aligned}
$$

Pour déterminer les valeurs numériques $c_{n}(-p)$, où $p$ est un positif entier, nous posons

$$
B_{p}^{n}=\sum_{s=1}^{s=i p-1}\left(\begin{array}{c}
p \\
s
\end{array}\right)(p-s)^{n}
$$

et nous aurons, en vertu de l'identité

l'expression suivante

$$
\left(\frac{1+e^{2 \tau}}{2}\right)^{p}=\frac{1}{2^{p}} \cdot \sum_{s=0}^{s=p}\left(\begin{array}{c}
p \\
s
\end{array}\right) e^{2(p-s) \alpha}
$$

$$
c_{n}(-p)=(-1)^{n} 2^{n-p} B_{p}^{n},
$$

de sorte que la formule générale (12) du paragraphe I donnera celte autre 
représentation indépendante

$$
c_{n}(x)=\sum_{p=1}^{p=n}(-1)^{n+p}\left(\begin{array}{c}
\frac{x}{q}+p-1 \\
p
\end{array}\right)\left(\begin{array}{l}
\frac{x}{q}+n \\
n-p
\end{array}\right)_{2^{n-1 q} B_{p q}^{n},}
$$

où $q$ est un positif entier quelconque.

Nous avons encore à considérer le développement suivant

$$
\left(\frac{1-e^{-\alpha}}{\alpha}\right)^{-x}=\sum_{n=0}^{n=\infty} \frac{d_{n}(x)}{n !} \alpha^{\alpha}, \quad|\alpha|<2 \pi
$$

multiplions par $\alpha^{-x}$ les deux membres de (12), puis différentions par rapport à $\alpha$, il en résulte l'équation fonctionelle

$$
x d_{n-1}(x)-x d_{n}(x+1)=(n-x) d_{n}(x)
$$

c'est-à-dire que nous aurons pour $n \geqq 1$

$$
d_{n}^{\prime}(0)=-\frac{d_{n}(1)}{n}, \quad d_{n-1}(1)=d_{n}(2)+(n-1) d_{n}(1)
$$

Cela posè, la série de puissances

$$
\frac{\alpha}{1-e^{-\alpha}}=1+\frac{\alpha}{2}+\sum_{n=1}^{n=\infty} \frac{(-1)^{n-1} B_{n}}{(2 n) !} \alpha^{2 n}, \quad|\%|<2 \pi,
$$

où les $B_{n}$ sont les nombres de Bernould, donnera les valeurs numériques suivantes

$$
\begin{aligned}
& d_{2 n}^{\prime}(0)=\frac{(-1)^{n}}{2 n} B_{n}, \quad d_{2 n+1}^{\prime}(0)=0 \\
& d_{2 n}(1)=(-1)^{n-1} B_{n}, \quad d_{2 n+1}(1)=0 \\
& d_{2 n}(2)=(-1)^{n}(2 n-1) B_{n}, \quad d_{2 n+1}(2)=(-1)^{n-1} B_{n} .
\end{aligned}
$$

Dans ces formules il faut admettre généralement $n \geqq 1$; mais l'expression de $d_{2 n}$ (2) n'est pas appliquable pour $n=1$; dans cette expression il faut supposer $n \geqq 2$.

Appliquons ensuite l'identité

$$
\left(\frac{1-e^{-\alpha}}{\alpha}\right)^{\prime}=\frac{(-1)^{p}}{\alpha^{p}} \sum_{s=0}^{\Sigma=p}(-1)^{s}\left(\begin{array}{c}
p \\
s
\end{array}\right) e^{-(p-s) \alpha},
$$

où $p$ est un positif entier, nous aurons en vertu de la définition (7) du pa- 
ragraphe IV la série de puissances toujours convergente

$$
\left(\frac{1-e^{-\alpha}}{\alpha}\right)^{p}=\sum_{n=0}^{n=\infty} \frac{(-1)^{n}}{(n+p) !} L_{p}^{n+\infty} \alpha^{n}
$$

ce qui donnera

$$
d_{n}(-p)=\frac{(-1)^{n} n !}{(n+p) !} I_{p}^{n+p}
$$

d'où la représentation indépendante

$$
d_{n}(x)=\sum_{p=1}^{p=n} \frac{(-1)^{n+\nu} n !}{(n+p q) !}\left(\begin{array}{c}
\frac{x}{q}+p-1 \\
p
\end{array}\right)\left(\begin{array}{l}
x \\
q \\
q \\
n-p
\end{array}\right) I_{p q}^{n+p q}
$$

où $q$ est un positif entier quelconque.

Remarquons en passant que les polynomes $d_{n}(x)$ sont intimément liés arec les fonctions de Strmuin(* $\left.{ }^{*}\right)$.

\section{TROISIEME PAR'TIE.}

\section{Table des formules numériques}

\section{FOHMUlas CONTENANT DHS NOMBRES QUelcionQUes.}

Désignons par $q$ un positif entier quelconque, tandis que $m$ est un entier qui doit satisfaire aux conditions indiquées, nous aurons les représentations indépendantes qui contiennent les deux nombres $m$ et $q$ :

$$
T_{n}=\sum_{p=1}^{p=m} \frac{(-1)^{n+1}}{p q \cdot 2^{2 x-1}}\left(\begin{array}{c}
m \\
p
\end{array}\right) \mathfrak{A}_{p q}^{\mathfrak{2} n}, \quad m \geqq n
$$

(*) Voir mon Handbuch der Theorie des Gammafunktion, p. 71-77; Leipsic, 1906. 


$$
\begin{aligned}
& E_{n}=\sum_{p=1}^{p=m} \frac{(-1)^{n+p}}{2^{p q-1}}\left(\begin{array}{c}
\frac{1}{q}+p-1 \\
p
\end{array}\right)\left(\begin{array}{c}
\frac{1}{q}+m \\
m-p
\end{array}\right)_{p q}^{2 n}, \quad m \geqq n . \\
& T_{n+1}=\sum_{p=1}^{p=m} \frac{(-1)^{n+p}}{2^{m-1}}\left(\begin{array}{c}
\frac{2}{q}+p-1 \\
p
\end{array}\right)\left(\begin{array}{c}
\frac{2}{q}+m \\
m-p
\end{array}\right) \mathfrak{M}_{p q}^{n, n}, \quad m \geqq n . \\
& \frac{2^{2 n} B_{n}}{2 n}=\sum_{p=1}^{p=m} \frac{(-1)^{n+p}(2 n) !}{p q \cdot(2 n+p q) ! 2^{p q-1}}\left(\begin{array}{c}
m \\
p
\end{array}\right) A_{p q}^{2 n+p q}, \quad m \geqq n . \\
& \left(2^{2 n}-2\right) B_{n}=\sum_{p=1}^{p=m} \frac{(-1)^{n+p}(2 n) !}{(2 n+p q) ! 2^{p q-1}}\left(\begin{array}{c}
\frac{1}{q}+p-1 \\
p
\end{array}\right)\left(\begin{array}{c}
\frac{1}{q}+m \\
m-p
\end{array}\right) A_{p q}^{2 n+p q}, \quad m \geq n \\
& (2 n-1) 2^{2 n} B_{n}=\sum_{p=1}^{p=m} \frac{(-1)^{n+p}(2 n) !}{(2 n+p q) ! 2^{n+-1}}\left(\begin{array}{c}
\frac{2}{q}+p-1 \\
p
\end{array}\right)\left(\begin{array}{c}
\frac{2}{q}+m \\
m-p
\end{array}\right) A_{p q}^{2 n+m^{m}}, \quad m \geqq n . \\
& T_{n}=\sum_{p=1}^{p=m} \frac{(-1)^{n+p} 2^{2 n-2 / q}}{p q}\left(\begin{array}{c}
m \\
p
\end{array}\right) B_{p q}^{2 n}, \quad m \geqq 2 n . \\
& T_{n+1}=\sum_{p=1}^{n=m}(-1)^{n+n+1} 2^{2 n-p q+1}\left(\begin{array}{c}
\frac{1}{q}+p-1 \\
p
\end{array}\right)\left(\begin{array}{c}
\frac{1}{q}+m \\
m-p
\end{array}\right) B_{p q}^{2 n+1}, \quad m \geqq 2 n+1 . \\
& T_{n+1}=\sum_{p=1}^{n=m}(-1)^{n+p+1} 2^{2 n-1, q}\left(\begin{array}{c}
\frac{2}{q}+p-1 \\
p
\end{array}\right)\left(\begin{array}{c}
\frac{2}{q}+m \\
m-p
\end{array}\right) B_{p q}^{2 n}, \quad m \geqq 2 n . \\
& 2 T_{n+1}=\sum_{p=1}^{p=m}(-1)^{n+p+1} 2^{2 n-p q+1}\left(\begin{array}{c}
\frac{2}{q}+p-1 \\
p
\end{array}\right)\left(\begin{array}{c}
\frac{2}{q}+m \\
m-p
\end{array}\right) B_{p q}^{2 n+1}, \quad n \geq 2 n+1 . \\
& \frac{B_{n}}{2 n}=\sum_{p=1}^{n=m} \frac{(-1)^{n+p}(2 n) !}{(2 n+p q) ! p^{\prime} q}\left(\begin{array}{c}
m \\
p
\end{array}\right) L_{p q}^{2 n+2 n q}, \quad m \geqq 2 n . \\
& B_{n}=\sum_{p=1}^{p=n} \frac{(-1)^{n+n+1}(2 n) !}{(2 n+p q) !}\left(\begin{array}{c}
\frac{1}{q}+p-1 \\
p
\end{array}\right)\left(\begin{array}{c}
\frac{1}{q}+m \\
m-p
\end{array}\right) L_{p q}^{m n+m,}, \quad m \geqq 2 n . \\
& (2 n-1) B_{n}=\sum_{p=1}^{i=m} \frac{(-1)^{n+p}(2 n) !}{(2 n+p q) !}\left(\begin{array}{c}
\frac{2}{q}+p-1 \\
p
\end{array}\right)\left(\begin{array}{c}
\frac{2}{q}+m \\
m-p
\end{array}\right) L_{p q}^{q n+p q}, \quad n \geqq 2 n \text {. } \\
& B_{n}=\sum_{p=1}^{p=n} \frac{(-1)^{n+p+1}(2 n+1) !}{(2 n+p q+1) !}\left(\begin{array}{c}
\frac{2}{q}+p-1 \\
p
\end{array}\right)\left(\begin{array}{c}
\frac{2}{q}+m \\
m-p
\end{array}\right) I_{m p}^{m p+p !+1} \cdot m \geqq 2 n+1 .
\end{aligned}
$$


Remplaçons maintenant toujours $m$ par sa plus petite valeur, puis posons $q=1$, et posons encore dans (3), (6), (9) et (10), (13) et (14) $q=2$, nous aurons les formules plus particulières et plus simples

I.

$$
T_{n}=\sum_{p=1}^{p=n} \frac{(-1)^{n+1}}{p \cdot 2^{n-1}}\left(\begin{array}{l}
n \\
p
\end{array}\right) \mathfrak{U}_{p}^{2 n} .
$$

II.

$$
E_{n}=\sum_{p=1}^{l=n} \frac{(-1)^{n+1}}{2^{p-1}}\left(\begin{array}{l}
n+1 \\
n-p
\end{array}\right) \mathfrak{M}_{p}^{3 n}
$$

III.

$$
T_{n+1}=\sum_{p=1}^{p=1} \frac{(-1)^{n+p}(p+1)}{2^{n-1}}\left(\begin{array}{l}
n+\frac{9}{n+p} \\
n-p
\end{array}\right) \mathfrak{F}_{p}^{2 n} .
$$

IV.

$$
T_{n+1}=\sum_{p=1}^{p=11} \frac{(-1)^{n+p}}{2^{2 p-1}} \cdot\left(\begin{array}{l}
n+1 \\
n-p
\end{array}\right) \mathfrak{W}_{2, p}^{i n} .
$$

V.

$$
\frac{2^{2 n} B_{n}}{2 n}=\sum_{i=1}^{n=1} \frac{(-1)^{n+p}(2 n) !}{(p+2 n) ! p \cdot 2^{p-1}}\left(\begin{array}{l}
n \\
p
\end{array}\right) d_{p}^{2 n+1} \text {. }
$$

VI.

$$
\left(2^{2 n}-2\right) B_{n}=\sum_{x=1}^{p=n} \frac{(-1)^{n+p}(2 n) !}{(p+2 n) ! 2^{p-1}}\left(\begin{array}{l}
n+1 \\
n-p
\end{array}\right) A_{p}^{2 n+p} \text {. }
$$

VII.

$$
(2 n-1) 2^{2 n} B_{n}=\sum_{p=1}^{p=n} \frac{(-1)^{n+p}(2 n) !(p+1)}{(p+2 n) ! 2^{p-1}}\left(\begin{array}{l}
n+2 \\
n-p
\end{array}\right) A_{p}^{2 n+p} \text {. }
$$

VIII.

$$
(2 n-1) 2^{2 n} B_{n}=\sum_{p=1}^{p=n} \frac{(-1)^{n+p}(2 n) !}{(2 p+2 n) ! 2^{2 p-1}}\left(\begin{array}{l}
n+1 \\
n-p
\end{array}\right) A_{2 p}^{2 n+2^{2}} \text {. }
$$

IX.

$$
T_{n}=\sum_{p=1}^{p=n_{n}^{n}} \frac{(-1)^{n+p} 2^{2 n-y^{2}}}{p}\left(\begin{array}{c}
2 n \\
p
\end{array}\right) B_{p}^{2 n} \text {. }
$$

$\mathrm{X}$.

$$
T_{n+1}=\sum_{p=1}^{p=2 n+1}(-1)^{n+p+1} 2^{2 n-p+1}\left(\begin{array}{l}
2 n+1 \\
2 n-p
\end{array}\right) B_{p}^{2 n+1}
$$

XI.

$$
T_{n+1}=\sum_{p=1}^{p=2 n}(-1)^{n+p+1} 2^{2 n-p}\left(\begin{array}{l}
2 n+2 \\
2 n-p
\end{array}\right)(p+1) B_{p^{2}}^{2 n}
$$

XII.

$$
2 T_{n+1}=\sum_{p=1}^{p=2 n+1}(-1)^{n+p+1} 2^{2 n-p+1}\left(\begin{array}{c}
2 n+3 \\
2 n-p+1
\end{array}\right)(p+1) B_{p}^{2 n+1}
$$

XIII.

$$
T_{n+1}=\sum_{p=1}^{p=2 n}(-1)^{n+p+1} 2^{2 n-2_{p} p}\left(\begin{array}{l}
2 n+1 \\
2 n-p
\end{array}\right) B_{2 p}^{2 n} \text {. }
$$

XIV.

$$
2 T_{n+1}=\sum_{p=1}^{p=2 n+1}(-1)^{n+p+1} 2^{2 n-2 p+1}\left(\begin{array}{c}
2 n+2 \\
2 n-p+1
\end{array}\right) B_{2 p}^{2 n+1} .
$$


XV.

$$
\frac{B_{n}}{2 n}=\sum_{p=1}^{p=2 n} \frac{(-1)^{n+p}(2 n) !}{(2 n+p) ! p}\left(\begin{array}{c}
2 n \\
p
\end{array}\right) L_{p}^{2 n+p}
$$

XVI.

$$
B_{n}=\sum_{p=1}^{p=2 n} \frac{(-1)^{n+1+1}(2 n) !}{(2 n+p) !}\left(\begin{array}{l}
2 n+1 \\
2 n-p
\end{array}\right) L_{p}^{2 n+1}
$$

XVII. $\quad(2 n-1) B_{n}=\sum_{p=1}^{p=2 n} \frac{(-1)^{n+p}(2 n) !(p+1)}{(2 n+p) !}\left(\begin{array}{l}2 n+2 \\ 2 n-p\end{array}\right) L_{p}^{2 n+p}$.

XVIII. $\quad B_{n}=\sum_{p=1}^{p=2 n+1} \frac{(-1)^{n+p+1}(2 n+1) !(p+1)}{(2 n+p+1) !}\left(\begin{array}{c}2 n+3 \\ 2 n-p+1\end{array}\right) L_{p}^{2 n+p+1}$.

XIX. $\quad(2 n-1) B_{n}=\sum_{p=1}^{p=1} \frac{(-1)^{n+1}(2 n) !}{(2 n+2 p) !}\left(\begin{array}{l}2 n+1 \\ 2 n-p\end{array}\right) L_{2 p}^{2 n+2 x^{\prime}}$.

XX. $\quad B_{n}=\sum_{p=1}^{i=1} \frac{(-1)^{n+p+1}(2 n+1) !}{(2 n+2 p+1) !}\left(\begin{array}{c}2 n+2 \\ 2 n-p+1\end{array}\right) L_{2 p}^{2 n+2 p+1}$.

Dans ces formules nous avons posé pour abréger

$$
\begin{array}{rlrl}
\mathfrak{Q}_{p}^{n} & =\sum_{s=0}^{\leqq \frac{p-1}{2}}\left(\begin{array}{c}
p \\
s
\end{array}\right)(p-2 s)^{n}, & A_{p}^{n}=\sum_{s=9}^{\leq \frac{p-1}{2}}(-1)^{s}\left(\begin{array}{c}
p \\
s
\end{array}\right)(p-2 s)^{n}, \\
L_{p}^{n}=\sum_{s=0}^{s=p-1}(-1)^{s}\left(\begin{array}{c}
p \\
s
\end{array}\right)(p-s)^{n}, & B_{p}^{n}=\sum_{s=0}^{s=p-1}\left(\begin{array}{c}
p \\
s
\end{array}\right)(p-s)^{\prime \prime} .
\end{array}
$$

De plus, nous avons entres les $T_{n}$ et les $B_{n}$ la relation suivante

$$
T_{n}=\frac{2^{2 n}\left(2^{2 n}-1\right)}{2 n} B_{n} .
$$

\section{VIIL. Représentatong sans des paramètres.}

Les représentations indépendantes d'une forme plus simple que nous venons d'indiquer pour les fonctions $a_{n}(x)$ et $c_{n}(x)$ donnent une suite d'autres formules numériques, savoir:

XXI.

$$
T_{n}=\sum_{p=1}^{p=\mathbb{Z}_{n}} \frac{(-1)^{n-1} 2^{2 n-p}}{p} I_{p}^{2 n}
$$

XXII.

$$
T_{n}=\sum_{p=1}^{p=\underline{\alpha}_{n-1}}(-1)^{n+2} 2^{2 n-p^{p-1}} L_{p}^{2 n-1}
$$




$$
\begin{aligned}
& \text { XXIII. } \\
& E_{n}=\sum_{p=0}^{p=\sum^{n} n} \frac{(-1)^{n+p}}{2^{p}} L_{p}^{2 n}(1) . \\
& \text { XXIV. } \\
& E_{n}=\sum_{p=1}^{p=0} \frac{(-1)^{n+1} p}{2^{p}} L_{p}^{2 n-1}(1) . \\
& \text { XXV. } \\
& T_{n+1}=\sum_{p=0}^{p=2 n} \frac{(-1)^{n+p}(p+1)}{2^{p}} I_{p}^{2 n}(2) . \\
& T_{n+1}=\sum_{p=1}^{p=2 n-1} \frac{(-1)^{n+p} \frac{p}{2^{p}}(p+1)}{2^{p}} I_{p}^{2 n-1}(2) . \\
& \mathcal{2}^{2 n} T_{n}=\sum_{p=1}^{p=n} \frac{(-1)^{n+p}}{2^{n-1} \cdot p} A_{2 p}^{2 n} \\
& \text { 2:" } E_{n}=\sum_{p=1}^{p=n} \frac{(--1)^{n-1}}{2^{p-1}} A_{2 p}^{2 n} \\
& 2^{2 n} T_{n+1}=\sum_{p=1}^{p=n} \frac{(-1)^{n+y^{n}}(p+1)}{2^{p-1}} A_{2 p}^{3 n} \text {. } \\
& T_{n}=\sum_{p=1}^{p=1} \frac{(-1)^{n+p}}{p \cdot 2^{2 n}} A_{2 p}^{2 n} \\
& E_{n}=\sum_{p=1}^{p=n} \frac{(-1)^{n+1}}{2^{2 p-1}}\left(\begin{array}{c}
p-\frac{1}{2} \\
p
\end{array}\right)_{A_{2,}^{2 n} \text {. }}^{2 n} \\
& T_{n+1}=\sum_{p=1}^{p=n} \frac{(-1)^{n+p}}{2^{2_{2}-1}} A_{2 p}^{2 n}, \\
& T_{n+1}=\sum_{p=1}^{p=2 n n}(-1)^{n+p+1}(p+1) 2^{2 n-p^{n}} L_{p}^{2 n} . \\
& 2 T_{n+1}=\sum_{p=1}^{p=2 n+1}(-1)^{n+p+1}(p+1) 2^{2 n-p+1} L_{p}^{n+1} \text {. } \\
& T_{n}=\sum_{p=1}^{p=2 n}(-1)^{n+n} \mathfrak{R}_{p}^{2 n-1} . \\
& T_{n+1}=\sum_{p=1}^{p=2 n+1}(-1)^{n+p+1} \mathbb{R}_{p}^{2 n+1} \text {. } \\
& T_{n+1}=\sum_{p=1}^{p=2 n}(-1)^{n+p+1} \Re_{p}^{\Re^{n}}(2) \text {. } \\
& 2 T_{n+1}=\sum_{p=1}^{p=2 n+1}(-1)^{n+p^{n-1}} q_{p}^{2 n+1}(2) \text {. }
\end{aligned}
$$


$\mathrm{XXXIX}$

$$
T_{n+1}=\sum_{p=0}^{p=2 n}(-1)^{n+p} 2^{2 n-p} L_{p+1}^{n+n+}
$$

XL.

$$
T_{n+1}=\mathbb{R}_{n+1}^{2 n+1}-2 \cdot \sum_{p=0}^{p=n-1}(-1)^{p} \mathbb{R}_{n-p}^{n+n+1}
$$

Les deux derniers développements sont tirés des formules (14) et (18) du paragraphe $V$.

Dans les formules de XXIII à XXVI il faut poser

$$
I_{p}^{n}(\alpha)=\sum_{s=0}^{s=p}(-1)^{\star}\left(\begin{array}{l}
p \\
s
\end{array}\right)(\alpha+2 p-2 s)^{n}, \quad L_{0}^{n}(\alpha)=\alpha^{\prime \prime},
$$

ce qui donnera

$$
L_{p}^{n}(0)=2^{n} \cdot L_{p}^{n}
$$

Quant à la fonction $\ell_{p}^{n}(x)$ qui figure dans les formules XXXVIl et XXXVIII, nous avons

$$
\mathfrak{R}_{p}^{n}(x)=\sum_{s=0}^{s=p-1}(-1)^{s}\left(\begin{array}{c}
x+p-s-1 \\
p-s
\end{array}\right)\left(\begin{array}{c}
x+n \\
s
\end{array}\right)(p-s)^{\prime \prime}
$$

ce qui donnera

$$
\left(D_{x} \ell_{p}^{n}(x)\right)_{x=0}=\ell_{p}^{n-1}(1)=\ell_{p}^{n-1}
$$

On voit que plusieurs des quarante représentations indépendantes particulières que nous venons d'indiquer sont bien connues. 